\title{
Los lenguajes de la inteligencia artificial, los lenguajes de la metafísica \\ y los lenguajes de la fe
}

The languages of artificial intelligence, the languages of metaphysics, and the languages of faith

\author{
JAVIER LEACH \\ Departamento de Sistemas Informáticos y Computación \\ Universidad Complutense de Madrid \\ leach@sip.ucm.es
}

Resumen. La formalización de los lenguajes de la matemática ha dado lugar a los lenguajes de la informática y de la inteligencia artificial. En este artículo estudio algunas de las propiedades semánticas de los lenguajes formales de la inteligencia artificial que llamo lenguajes del signo. Comparo y contrapongo las propiedades semánticas de estos lenguajes formales de la inteligencia artificial con la semántica más amplia de los lenguajes humanos de la metafísica y la fe religiosa, que llamo lenguajes del símbolo.

Palabras clave: lenguajes formales del signo; lenguajes humanos del símbolo; inteligencia artificial; metafísica; fe religiosa.

Abstract. The formalization of the languages of mathematics has led to the formal languages of computer science and artificial intelligence. This article studies a few semantic properties of the formal languages of artificial intelligence, which I call languages of sign. This paper compares and opposes the semantic properties of these formal languages of artificial intelligence with the richer semantic properties of the languages of metaphysics and religious faith, which I call languages of symbol.

Keywords: formal languages of sign; symbol languages; artificial intelligence; metaphysics; religious faith. 


\section{Dos tipos básicos de lenguaje}

En el libro Matemáticas y Religión (Leach 2010), distingo dos tipos básicos de lenguaje que llamo lenguajes del signo y lenguajes del símbolo. Llamo lenguajes del signo a los lenguajes propios de la matemática y de las ciencias empíricas y llamo lenguajes del símbolo a los propios de la metafísica y la religión. En este artículo quiero comparar estos dos tipos de lenguaje para distinguirlos, a la vez que señalo algunas de sus mutuas interrelaciones. La motivación para estudiar y comparar estos dos tipos de lenguaje es, por una parte, la particular importancia que han adquirido actualmente los lenguajes que llamo del signo como lenguajes de la inteligencia artificial $\mathrm{y}$, por otra parte, la importancia humana que tienen los lenguajes que llamo del símbolo como medio de comunicación de experiencias específicamente humanas y religiosas.

\section{Inteligencia artificial}

El inventor y futurólogo Raymond Kurzweil predice que los ordenadores pasarán el Test de Turing en un futuro próximo; más en concreto predice que lo pasarán en el año 2029 (singularityhub 2011). El Test de Turing es una prueba de que la inteligencia artificial (IA) de un ordenador iguala o supera la inteligencia de una persona humana. En este test, un juez externo hace todo tipo de preguntas a un ordenador y a una persona humana, estando los dos aislados en dos habitaciones distintas. Si el juez no es capaz de distinguir por sus respuestas el ordenador de la persona humana, entonces el ordenador ha pasado la prueba de inteligencia. Para que la inteligencia de un ordenador supere o iguale la de una persona humana, las pruebas han de incluir las capacidades fundamentales no solo de inteligencia abstracta, sino también de autoconciencia, y las aptitudes emocionales propias de la persona humana. Actualmente hay robots, que algunos llaman humanoides, que muestran algunas de estas capacidades como la autoconciencia que proviene del conocimiento de su propio programa y del conocimiento de su propia actividad informática, junto con una cierta aptitud para percibir emociones humanas y una disposición para 
reaccionar con empatía ante las emociones humanas. Basándose en estas capacidades, los defensores de la IA fuerte opinan que los ordenadores serán en un futuro indistinguibles de los humanos y que se llegará a un punto singular en la evolución humana en la que aparecerá un nuevo tipo post-humano fruto de la interacción entre humanos y robots.

\section{La habitación China}

El filósofo John Searle representa una visión menos optimista acerca de las capacidades futuras de un ordenador. Searle opina que los ordenadores se diferencian de los humanos porque no tienen conciencia como tenemos las personas humanas. En su libro Mentes, Cerebros, Ciencia (Searle 1984) Searle usa el experimento mental llamado "La habitación China" para mostrar que un ordenador realiza acciones sin tener conciencia de lo que está haciendo, mientras que los humanos tenemos conciencia de nuestras acciones. En su experimento Searle supone que un ordenador ha pasado el Test de Turing comparando sus capacidades con las de una persona humana que habla chino. Entonces Searle se introduce él mismo, que es consciente de no saber chino, en la habitación en la que estaba el ordenador y usa los medios de los que dispone el ordenador y, bajo esas condiciones, el juez no es capaz de distinguir a Searle de la persona que habla chino. Luego, concluye Searle, el ordenador no necesita ser consciente de saber chino para pasar el test de La Habitación China, basta con que realice la acción de hablar chino, sin ser consciente de que ha realizado esa acción.

\section{IA fuerte}

Los detractores de Searle defienden lo que algunos llaman la IA fuerte, es decir la IA de las máquinas que abarca todas las capacidades cognoscitivas del ser humano, incluidas la conciencia y las capacidades emotivas. Para sus detractores, el hecho de que Searle tenga conciencia de no saber chino no prueba que la máquina no pueda tener conciencia de saber chino. Para estos, el ordenador que utiliza Searle para engañar al juez puede ser consciente de que sabe chino aunque Searle sea consciente de no saber chino. 


\section{Dos tipos de lenguaje y dos tipos de conciencia}

En este artículo quiero distinguir entre la conciencia mecánica que puede tener un ordenador de la conciencia humana, y establecer una conexión entre los distintos tipos de conciencia y los distintos tipos de lenguaje. Apoyándome en esa conexión entre conciencia y lenguaje, quiero defender la tesis de que los lenguajes que usa un ordenador sólo pueden comunicar el estado de un cierto tipo de conciencia que no incluye todas las características de la conciencia propia del ser humano. Más en concreto distinguiré entre la conciencia que puede tener un ordenador y la conciencia humana basándome en la distinción entre los lenguajes del signo y los lenguajes del símbolo a los que hacía referencia al comienzo del artículo.

La conciencia del ordenador no es sino el conocimiento que tiene el ordenador de su propio programa, de sus propias acciones informáticas y el conocimiento que tiene de la adecuación de sus acciones a su programa. Esa conciencia del ordenador se comunica mediante el lenguaje que yo llamo del signo en el que está escrito el programa del ordenador. Como veremos, la conciencia humana no se puede comunicar con el lenguaje del signo por ser una experiencia personal de libertad compartida comunitariamente con otros seres humanos.

Mantendré en este artículo que mientras los lenguajes del signo son básicos y los compartimos los humanos con los ordenadores, los lenguajes del símbolo tienen un significado que va más allá de lo puramente mecánico; son específicamente humanos y su semántica es específicamente humana. El lenguaje con el que los humanos comunicamos con otros humanos la experiencia de nuestra conciencia pertenece a los lenguajes del símbolo y es distinto del lenguaje con el que el ordenador comunica la experiencia de su conciencia.

\section{Lenguaje, libertad, confianza}

En el experimento de La Habitación China, Searle es consciente de no saber chino pero opta libremente por engañarnos. Sabiendo que no sabe chino, Searle opta por engañarnos usando los medios propios del ordenador, 
y luego nos comunica que nos ha engañado diciéndonos que es consciente de no saber chino. Nosotros creemos a Searle y por eso tiene sentido el experimento de La Habitación China. Cuando Searle nos explica su experimento mental y nos comunica que no sabe chino todos entendemos que Searle hace una opción libre, que no se trata de una mera decisión mecánica (Leach 2012).

\section{La confianza en el ordenador y la confianza humana}

Las pruebas de Turing y de La Habitación China muestran, a mi modo de ver, distintos tipos de confianza en la verdad de los enunciados de un lenguaje. No es lo mismo confiar en la palabra de un ordenador que confiar en la palabra de una persona humana. La confianza en la palabra de un ordenador es una acción mecánica basada en las reglas mecánicas propias de los lenguajes en los que el ordenador está programado. La confianza en la palabra de una persona humana está basada en nuestra relación personal con ella.

\section{La verificación de un programa}

El máximo grado de confianza en los enunciados de un ordenador lo logramos cuando verificamos su programa. La verificación de un programa de ordenador consiste en demostrar matemáticamente que el programa ejecuta las acciones para las cuales ha sido programado. La verificación de un programa es un proceso matemático difícil y, generalmente, solo se logra verificar programas sencillos. Si un programa está verificado confiamos totalmente en las acciones que realiza el ordenador siguiendo el programa.

Cualquier error que tenga un ordenador cuyo programa esté matemáticamente verificado sabemos que no puede provenir del programa; podrá provenir del hardware o será un error del usuario. La confianza en un programa verificado es la confianza que tenemos en un siervo que sabemos que no nos engañará y que ejecutará con precisión las órdenes que le hemos dado, de acuerdo con su naturaleza y la capacidad que tenga de ejecutarlas. Los aviones, los trenes, los sistemas de semáforos que regulan el tráfico, 
todos ellos están regulados por ordenadores, y frente a todos ellos tenemos una confianza basada en su funcionamiento mecánico.

\section{Niveles en la confianza}

Sin embargo, los lenguajes humanos trasmiten otro tipo de confianza o desconfianza más profundo que la confianza o desconfianza que pueden trasmitir los lenguajes de programación. La distinción entre lenguaje del signo y lenguaje del símbolo puede ayudarnos en la discusión acerca de dos niveles de confianza en el lenguaje humano, el nivel mecánico y el nivel específicamente humano. Podemos tener distintos tipos de confianza en la naturaleza humana. Cuando confiamos en otra persona podemos hacerlo porque sabemos que será fiel a reglas mecánicas, igual que es fiel un robot o un drone en un campo de batalla; o podemos confiar más profundamente en una persona porque sabemos que ante la indeterminación mecánica reaccionará movido por la ley interior del amor. En el nivel mecánico del lenguaje del signo encontramos razones científicas para confiar en la naturaleza humana. Pero no podemos separar el conocimiento meramente científico de la naturaleza humana, que estudia el comportamiento de la naturaleza humana de acuerdo con reglas que la acercan al comportamiento de la máquina, del conocimiento específicamente humano de la naturaleza humana que expresamos mediante el lenguaje del símbolo.

\section{Semántica pública y semántica personal}

Los lenguajes del signo se caracterizan sobre todo porque sus semánticas son públicas, es decir, buscan tener siempre y bajo cualquier circunstancia el mismo significado para todos. Técnicamente, los matemáticos y los informáticos expresan el carácter público de estos lenguajes describiendo el significado de sus enunciados mediante modelos semánticos formales que, en cualquier cultura, en cualquier situación humana y en cualquier contexto religioso, pretenden tener siempre el mismo significado. Los lenguajes de programación de la informática son ejemplos de lenguajes del signo que últimamente han cobrado gran importancia cultural. 
Por ejemplo, el enunciado dos más dos son cuatro pertenece al lenguaje del signo, porque cuando digo que dos más dos son cuatro estoy expresando un enunciado que pretende ser entendido con el mismo significado en cualquier situación humana. Los matemáticos describen la semántica de este enunciado de diversas formas; por ejemplo, mediante conjuntos de números. En la teoría de conjuntos describen cualquier objeto en general como el conjunto de sus elementos y definen su significado semántico con claridad de modo que permanezca igual, siempre y en cualquier circunstancia humana.

Cuando Searle dice que no sabe chino la semántica de su enunciado es personal y yo juzgaré que ese enunciado es verdadero porque confío en la palabra de Searle.

\section{Los lenguajes y la metafísica (Leach 2014)}

En el libro Matemáticas y Religión mantengo que las ideas metafísicas y religiosas se expresan con otro tipo de lenguajes que llamo lenguajes del símbolo. Los lenguajes del símbolo los distingo de los lenguajes del signo por el carácter personal de su semántica. La semántica personal de los lenguajes del símbolo excede la mera semántica formal de los lenguajes del signo porque el significado de los lenguajes del símbolo está ligado a actitudes y opciones que son personales y comunitarias.

Por ejemplo, cuando digo que Dios es el creador del Universo, el significado de ese enunciado no será el mismo para un creyente que para un no creyente. El hecho de aceptar o de rechazar el significado de ese enunciado como verdadero, incluye un proceso de transformación personal que afecta a las relaciones del creyente, o del no creyente, con las realidades creadas del Universo y con el creador de todas ellas. En ese proceso personal, el hecho de que el creyente trate a las distintas realidades del Universo como creadas afecta a su relación personal con Dios como creador de todas ellas.

\section{Semánticas no simétricas}

En el libro Matemáticas y Religión planteo la relación entre los lenguajes del signo y los lenguajes del símbolo como asimétrica, porque se trata de 
dos visiones de una misma realidad pero desde perspectivas distintas. Aquí quiero insistir en el aspecto semántico de esa asimetría. Semánticamente estos lenguajes no son simétricos porque el significado semántico de los lenguajes del símbolo excede, va más allá, del significado semántico de los lenguajes del signo. Ese "exceso" proviene del carácter personal de la semántica de los lenguajes del símbolo. Tal exceso es importante en nuestra cultura informática porque, mientras que por una parte la Inteligencia Artificial nos permite traducir cualquier lenguaje humano, ya sea metafísico o religioso, a lenguajes del signo, por otra parte, cuando traducimos los lenguajes del símbolo a lenguajes del signo, estamos reduciendo esencialmente aspectos personales de su significado semántico.

Podemos expresar esta asimetría diciendo que, si bien es verdad que todo símbolo puede ser tratado semánticamente como un signo, sin embargo al tratarlo como signo estamos limitando una parte esencial de su significado. Dicho con otras palabras: el significado del lenguaje del símbolo tiene aspectos objetivables que pueden ser tratados mediante el lenguaje del signo, pero tiene otros aspectos muy importantes cuyo significado depende de la libertad personal del que usa esos lenguajes y que, por lo tanto, no son objetivables.

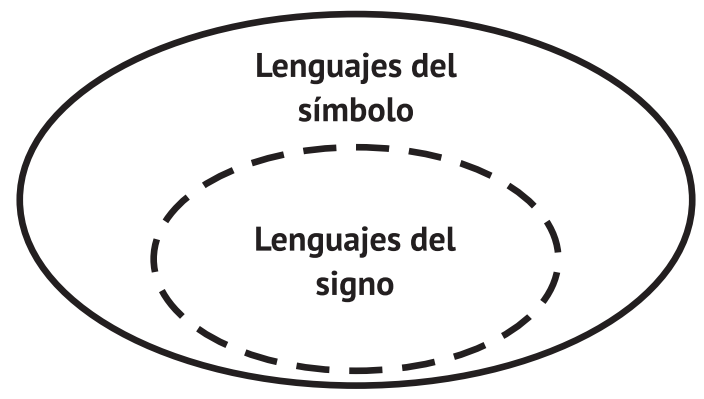

Figura 1. Relación entre los lenguajes del signo y del símbolo.

Esta situación la represento en la Figura 1 separando los lenguajes del signo de los lenguajes del símbolo por una línea de trazos no continua. La línea de 
trazos indica que la elipse de los lenguajes del signo puede crecer y abarcar a todos los lenguajes del símbolo, y así ocurre cuando los símbolos son tratados como signos por las máquinas en la Inteligencia Artificial. Por otra parte, la Figura 1 quiere indicar también el exceso de significado que hay en los lenguajes del símbolo. Dicho exceso hace que separemos ambos lenguajes, ya que si reducimos los lenguajes del símbolo a meros lenguajes del signo empobrecemos esencialmente el lenguaje humano.

\section{Historia de los lenguajes del signo}

Los lenguajes de las ciencias de la naturaleza no han sido siempre tal como los conocemos ahora. Aunque, sobre todo en la cultura occidental desde los tiempos de la civilización griega, los lenguajes de la ciencia han incluido ciertos lenguajes formales basados en reglas mecánicas de la lógica y la matemática, la relación entre los lenguajes de la ciencia y la matemática no ha sido siempre la misma. A lo largo de la historia, los lenguajes de la matemática se han ido transformando y han dado lugar a los lenguajes formales de la informática, que actualmente son uno de los principales instrumentos lingüísticos para el estudio de las ciencias de la naturaleza.

En el proceso histórico por el que hemos llegado al uso actual de los lenguajes formales en la informática, hay algunos momentos históricos que conviene señalar, porque en ellos se han ido desarrollando cuatro características que creo que especifican a los lenguajes actuales que yo llamo del signo:

1. Estructuración deductiva de los enunciados: formando teorías cuyos enunciados se deducen a partir de ciertos enunciados significados, llamados axiomas. Históricamente, en la Grecia de Pitágoras, Aristóteles y Euclides se comenzó a estructurar deductivamente el lenguaje científico.

2. Formulación en lenguaje matemático de las experiencias empíricas. Con la aparición de la Ciencia Moderna en el siglo XVII, científicos como Galileo, Newton y Leibniz dieron un paso definitivo al utilizar de un modo nuevo la matemática como lenguaje con el que expresar con precisión las observaciones científicas. 
3. Formalización del lenguaje matemático. En el siglo XIX, Gottlob Frege describió en el libro Begriffsschrift (Conceptografía) un lenguaje formal capaz de representar cualquier formulación y cualquier deducción matemática.

4. Descripción formal de los procesos mecánicos. En los años 30 del siglo XX, el inglés Alan Turing y el norteamericano Alonzo Church describieron formalmente los procesos mecánicos que puede ejecutar un ordenador mediante la máquina de Turing y el cálculo lambda, que resultaron ser equivalentes.

En este proceso histórico, los lenguajes del signo han ido adquiriendo progresivamente las cualidades características que poseen actualmente. Estas características han dado lugar a que, en algunos aspectos importantes, los lenguajes de la ciencia y la tecnología se aíslen de los lenguajes que llamo del símbolo. Uno de mis intenciones en este artículo es trazar puentes que conecten los lenguajes del signo propios de las ciencias de la naturaleza con los lenguajes del símbolo propios de la metafísica y la fe religiosa.

\section{La metamatemática y el programa de Hilbert}

El matemático alemán David Hilbert (1862-1943) llamó metamatemática al estudio con métodos matemáticos de las propiedades de la matemática. La metamatemática de Hilbert era posible porque la formalización del lenguaje matemático permitía tratar al lenguaje matemático como como objeto de estudio de la misma matemática.

El programa de Hilbert incluía la demostración metamatemática de ciertas propiedades de las teorías matemáticas. Entre esas propiedades fue especialmente importante la demostración de que las teorías matemáticas se podían deducir a partir de un conjunto finito y consistente de axiomas y de reglas formales de deducción. El programa de Hilbert cobró especial importancia en la primera mitad del siglo XX, pues la demostración matemática de la consistencia y la completitud de las teorías matemáticas permitían una confianza total en el valor semántico de dichas teorías. En el año 1932, el matemático Kurt Gödel demostró, mediante los teoremas de 
incompletitud de la aritmética, que el programa de Hilbert no era aplicable a la aritmética y, por lo tanto, no era aplicable a una parte muy importante de la matemática.

\section{Los ordenadores y la IA}

La inteligencia artificial (IA) es la inteligencia mediante la que podemos entender el lenguaje del signo. Alan Turing, el conocido informático teórico inglés dio una famosa conferencia el 20 de Febrero de 1947 en la London Mathematical Society. En esa conferencia dijo: "Se ha dicho que los equipos informáticos sólo pueden llevar a cabo los procesos para los que están programados. Esto es cierto en el sentido de que si hacen algo distinto de aquello para lo que están programados entonces cometen un error. También es cierto que la intención en la construcción de estas máquinas es, en primer lugar, que cumplan una tarea exacta, haciendo tan sólo los trabajos para los que han sido programadas, de tal modo que el usuario de la máquina puede, en principio, controlar completamente y en todo momento lo que está pasando. Hasta el presente sólo se han utilizado este tipo de máquinas.” (Carpenter and Doran 1986).

Esto lo decía Turing en el año 1947. Actualmente, ya hay máquinas que aprenden y se auto-educan modificando su propio programa. Turing, como profeta de los cambios que iban a ocurrir en la informática, continuó diciendo en su conferencia. "Supongamos que hemos creado una máquina con un cierto programa inicial, construido de forma que si se presentara una buena razón, el programa pudiera modificarse a sí mismo. Uno puede imaginar que después de que la máquina haya estado operando algún tiempo, puede ocurrir que el programa inicial se haya alterado de tal modo que ya no sea reconocible... En tal caso, se tendría que admitir un progreso de la máquina que no estaba previsto cuando se escribió el programa original. Sería como un alumno que ha aprendido mucho de su maestro, pero que ha añadido mucho más por su propio trabajo. Cuando esto sucede, creo que uno está obligado a considerar que la máquina es inteligente...” (Carpenter and Doran 1986). 
Las máquinas son inteligentes lo mismo que los seres humanos somos inteligentes, y hay un tipo de inteligencia que compartimos los humanos con las máquinas. Esa inteligencia, que nos permite comunicarnos en lenguajes formales del signo, tiene limitaciones radicales al mismo tiempo que posibilidades ilimitadas.

\section{Limitaciones y posibilidades de las decisiones mecánicas}

Hablemos primero de las limitaciones. Turing conocía perfectamente los teoremas de incompletitud de los sistemas formales y, en esa misma conferencia, dijo a continuación: "Yo diría que debemos de jugar limpio con la máquina, permitiéndole dar a veces respuestas incorrectas... En otras palabras, si se espera que una máquina sea infalible, entonces no puede ser también inteligente. Hay varios teoremas matemáticos que dicen casi exactamente eso.” (Carpenter and Doran 1986). Turing se refiere a la incompletitud de los sistemas formales y a la imposibilidad de determinar si una máquina arbitraria de Turing parará después de dar un número finito de pasos, con una palabra arbitraria dada como entrada. El tratamiento mecánico de la información tecnológica tiene límites para las máquinas y para los humanos.

Junto con esas limitaciones, el tratamiento mecánico de la información tecnológica puede crecer sin límites conocidos. Los sistemas informáticos son incompletos (Gödel 1931), pero pueden continuar creciendo y, efectivamente, continúan creciendo como pozos de conocimiento cuyo límite no conocemos. Turing nos lo recuerda en la misma conferencia cuando dice: “... estos teoremas no dicen nada acerca de cuánta inteligencia se puede mostrar una máquina si no tiene ninguna pretensión de infalibilidad." (Carpenter and Doran 1986). La capacidad de interacción entre la mente humana y el ordenador crece actualmente y, previsiblemente, continuará creciendo.

\section{La mente humana y el computador}

La mente humana es capaz de entender y comunicarse con los computadores. La revolución informática ha hecho que los seres humanos comparta- 
mos cada vez más con los ordenadores los lenguajes matemático-formales de la ciencia. Históricamente, los humanos utilizábamos lenguajes matemático-formales ya antes de la revolución informática. La revolución informática también ha mostrado que, en algunos aspectos, los ordenadores usan mejor esos lenguajes que nosotros los humanos. Tienen por ejemplo mayor capacidad de cálculo.

Como consecuencia del hecho de compartir con los ordenadores el lenguaje matemático-formal, los humanos compartimos cada vez más nuestra actividad científica con los ordenadores.

Sin embargo, tal como me gustaría mostrar en este artículo, existen otros lenguajes humanos, cuyo significado va más allá de lo formal y que no podemos compartir con los ordenadores. Entre estos lenguajes cuyo significado va más allá de lo formal, están los lenguajes que usamos los humanos tanto para expresar nuestra búsqueda del último sentido - búsqueda que llamaré metafísica-como nuestra capacidad de escucha religiosa.

\section{Los lenguajes de la metafísica}

Llamo lenguajes de la metafísica a los lenguajes que utilizamos para responder a las preguntas abiertas que las ciencias empírico-matemáticas dejan sin respuesta. Las ciencias de la naturaleza plantean cuestiones metafísicas, pero no pueden responderlas.

La pluralidad de sistemas formales crece actualmente en número y en extensión. Sabemos que no podemos construir un sistema formal único que abarque a todos los sistemas y que permita controlarlos formalmente. Esta situación nos lleva a la afirmación metafísica de que el último control de los sistemas formales es externo a esos mismos sistemas formales. Necesitamos otros lenguajes no formales que nos permitan controlar los sistemas formales.

\section{Ejemplos de preguntas metafísicas que la ciencia deja abiertas}

Una primera pregunta que podemos considerar metafísica proviene de las limitaciones inherentes a los lenguajes formales. Estas limitaciones nos 
llevan a pensar que más allá de los lenguajes formales existe otra racionalidad. ¿Podemos hacer coincidir los límites de la racionalidad con los límites de nuestros lenguajes formales? Y si no podemos limitar de ese modo nuestra racionalidad, ¿en qué lenguaje podemos expresar la racionalidad no formal, externa a los lenguajes formales?

Otra segunda pregunta proviene de la creatividad en los lenguajes formales. Los lenguajes formales continúan creciendo y multiplicándose y no conocemos los límites de ese crecimiento. ¿Son los lenguajes formales un cáncer de nuestro pensamiento que crece sin control externo?

Además de estas, hay otras preguntas más directa y explícitamente metafísicas, que la ciencia no puede responder. Por ejemplo:

\section{a. ¿Por qué es la realidad inteligible?}

La ciencia asume que la realidad es inteligible y estudia las leyes que rigen la inteligibilidad de la realidad, pero deja abierta la pregunta acerca de por qué es la realidad inteligible. Algunas ciencias de la naturaleza, como la neurociencia, estudian las leyes causales de la inteligibilidad, pero todas ellas asumen como una hipótesis no demostrada que la realidad es inteligible. La metafísica se plantea la pregunta, ¿por qué es la realidad inteligible?

\section{b. ¿Por qué necesita el lenguaje ser consistente o para-consistente?}

La ciencia también presupone una cierta consistencia del lenguaje humano, ya que sin una cierta consistencia no puede haber pensamiento deductivo lógico. En los sistemas para-consistentes, podemos admitir enunciados contradictorios del tipo A y no A, pero debemos entonces restringir las reglas de deducción para que no podamos deducir cualquier enunciado. La ciencia necesita de la consistencia, pero deja abierta la pregunta de por qué el pensamiento humano es consistente. La ciencia acepta la necesidad de limitar la capacidad de deducción de los sistemas como un hecho de nuestro pensamiento. Pero la ciencia no explica por qué se produce este hecho.

\section{c. ¿Por qué merece la pena hacer lo bueno?}

La mirada empírico-matemática produce una información que no compromete éticamente con la realidad. El lenguaje del signo no compromete éti- 
camente. El lenguaje del signo permite desarrollar la tecnología mediante la cual podemos controlar técnicamente la realidad, pero no puede trasmitir una información que comprometa éticamente a la persona que controla la realidad. Para que una información comprometa éticamente, el sujeto que recibe la información necesita valorarla como buena o como mala.

\section{d. ¿Por qué hay algo?}

Esta es quizás la cuestión metafísica más radical. Para filósofos como Leibniz o Heidegger, esta es la cuestión central de la metafísica. Esta última pregunta no tiene una respuesta científica. La ciencia siempre presupone la existencia de la realidad. Presupone que hay algo.

\section{Las cuestiones metafísicas permanecen}

Las preguntas metafísicas sobre las razones últimas permanecen cuando sucesivamente encontramos nuevas respuestas científicas empírico-matemáticas, que van respondiendo parcialmente a nuestras preguntas sobre la naturaleza de las cosas.

\section{Los valores y las respuestas metafísicas}

Las respuestas a las preguntas metafísicas no son analíticas, son globales y afectan a la actitud personal hacia la realidad global del sujeto que contesta a estas preguntas. Existen diferentes posibles respuestas a las preguntas metafísicas. La semántica de los lenguajes metafísicos no es meramente formal, es personal y comunitaria. Es decir, incluye explícitamente, junto con aspectos objetivos matemático-formales, valores personales y comunitarios que solo se entienden desde los impulsos internos de quien usa esos lenguajes y que, por lo tanto, no son públicos del mismo modo como lo son los lenguajes formales.

Pero cuando miramos al mundo desde la religión es necesaria una apertura a la visión empírico-matemática del mundo. En esto mi visión es distinta de la de Ian Gould, antropólogo norteamericano que defiende que los magisterios que no se sobreponen. Ian Gould, que se declara agnósti- 
co, defiende que el magisterio de la religión tiene su ámbito propio en las cuestiones de la moral, mientras que el magisterio de la ciencia tiene un valor que no interfiere con el magisterio de la religión, pues no entra en las cuestiones de los valores y la moral. Mi visión es distinta y quiero insistir en que mientras la ciencia tiene una capacidad metodológica de encerrarse en sí misma, sin embargo, la religión no puede encerrarse en sí misma, necesita abrirse a la ciencia empírica y a la matemática.

La mirada de la ciencia deja abiertas preguntas humanas. La mirada metafísica permite dar respuesta a preguntas que la ciencia deja abiertas y cuyo significado compromete personalmente al que las responde. A través del conocimiento que trata de ser objetivo, separado de nosotros, y que intenta aislarse en un lenguaje de signos, descubrimos el conocimiento simbólico que se caracteriza porque no podemos entender su significado si no hay en nosotros una transformación personal.

\section{Los lenguajes y la fe religiosa}

\section{La acción de Dios}

Las religiones abrahámicas van más allá de la metafísica, del conocimiento meramente metafísico que puede implicar la existencia de un Dios lejano y trascendente. En ellas, el Dios trascendente al Universo actúa, se hace presente y entra en diálogo con los hombres y mujeres, y ese diálogo trasforma los valores y las valoraciones del sujeto humano y a la comunidad humana que escucha la Palabra de Dios.

\section{La Encarnación}

En el cristianismo, la acción de Dios se manifiesta por la Palabra. El Dios místico y trascendente de la metafísica se manifiesta al mundo por la Encarnación del Hijo. Por la Encarnación, la mirada religiosa al mundo no es ajena a ninguna de las realidades mundanas e incluye tanto la mirada metafísica como la empírico-matemática, integrando las informaciones y las valoraciones del signo y del símbolo. 
En la tradición de los Ejercicios Espirituales de San Ignacio, a la que yo pertenezco, la contemplación religiosa del mundo lleva a amar a Dios en todas las cosas y a amar a todas las cosas en Dios.

\section{La ética y las religiones}

La ética usa lenguajes que son puente de unión entre la metafísica y las religiones. Ambas, la metafísica y las religiones, hablan del bien y del mal éticos. Las dos hablan del trabajo como servicio, de la misericordia y del compromiso con los débiles y su valor ético.

\section{Palabras y silencio}

Puede parecer paradójico que haya empezado hablando del lenguaje y termine hablando del silencio. Pero el silencio y la escucha no se pueden separar de la palabra, pues sin la escucha que se da en el silencio no hay ningún tipo de conocimiento y ningún tipo de palabra; tampoco hay conocimiento empírico ni lenguaje matemático-formal.

Pero el silencio es especialmente importante en la religión, porque en la religión se realiza el encuentro entre la acción del Dios trascendente y las palabras humanas con significado inmanente.

El diálogo con el Dios trascendente es necesariamente global. No puede limitarse a un aspecto o una parte de la realidad, porque Dios dejaría de ser trascendente y se relacionaría sólo con una parte de la realidad.

El silencio religioso es un silencio total. No hay palabra sin silencio, pero en el caso de la religión el silencio adquiere una importancia total. El silencio religioso nos permite entrar en conversación con el Dios trascendente, valorando internamente el significado de las palabras y dándoles a todas un significado global en Dios. Toda palabra puede, desde el silencio, cobrar un significado religioso.

\section{La mirada científica de la realidad y las miradas metafísica y religiosa}

Cuando hablamos del conocimiento metafísico y del conocimiento religioso, no tratamos de confiar poco o confiar menos en la ciencia, tratamos 
de confiar totalmente en la ciencia abierta que deja un espacio para la búsqueda metafísica y para la escucha de la fe. La mirada de la ciencia es poderosa tecnológicamente, es capaz de transformar el mundo pero no es capaz de interpretarlo. La mirada religiosa desde la escucha y el silencio no puede cerrarse sobre sí misma, como mera mirada científica y tecnológica; necesita abrirse al mundo científico y tecnológico entendiéndolo como servicio. Porque el servicio ético, que nace de la metafísica y la religión, pasa necesariamente por el mundo científico y tecnológico.

\section{Referencias}

Carpenter, B.E., and R.W. Doran (eds.). 1986. A. M. Turing's ACE Report of 1946 and Other Papers. Vol. 10 of Charles Babbage Institute Reprint Series for the History of Computing. Cambridge, MA: MIT Press.

Gödel, K. 1931. "Über formal unentscheidbare Sätze der Principia Mathematica und verwandter Systeme." Monatshefte für Mathematik und Physik 38: 173-198.

Kurzweil, R. 2011. "Kurzweil is confident machines will pass Turing Test by 2029." Accessed February 28, 2014. http://singularityhub.com/2011/04/04/kurzweil-is-confident-machines-will-pass-turing-test-by-2029-video-2/.

Leach, J. 2010. Mathematics and Religion. West Conshohocken, Pa: Templeton Press. [Traducción española: 2011. Matemáticas y Religión. Madrid: Sal Terrae.]

-. 2012 "Taking Options and Decisions." Revista Portuguesa de Filosofía 68/1-2: 87-104.

-. 2014 “I linguaggi della scienza, della metafisica e della religione.” La Civiltà Cattolica (to be published).

Searle, J. 1984. Minds, Brains and Science. Harvard: Harvard Univ Press. [Traducción española: 2001. Mentes, Cerebros, Ciencia. Madrid: Cátedra.] 